\title{
Colloidal Oatmeal Cream
}

National Cancer Institute

\section{Source}

National Cancer Institute. Colloidal Oatmeal Cream. NCI Thesaurus. Code C97918.

A colloidal oatmeal-based skin cream with potential moisturizing and skin protecting activity. Upon application to the skin, colloidal oatmeal cream forms a protective barrier and thereby prevents water loss, provides moisture to the skin and protects the skin from damage. 